\title{
High-sensitivity, wide-dynamic-range avalanche photodiode pixel design for large-scale imaging arrays
}

\author{
Alvin G. Stern \\ Boston University \\ Department of Electrical Engineering \\ 8 St. Mary's Street \\ Boston, Massachusetts 02215 \\ ag_stern@hotmail.com \\ Daniel C. Cole \\ Boston University \\ Department of Mechanical Engineering \\ 15 St. Mary's Street \\ Brookline, Massachusetts 02446
}

\begin{abstract}
We present a detailed design study for a novel solidstate focal plane array of silicon avalanche photodiodes (APDs), with each detector in the array capable of operating with wide dynamic range in linear or Geiger-mode. The detector array is based on back-illuminated, crystallographically etched, (100) epitaxial silicon on R-plane sapphire substrates using $27 \mu \mathrm{m}$ pixels, although other sizes could be supported as well. Analysis of the APD pixel design, which includes the photoelectron noise, dark current noise, and electronic circuit noise, shows the capability of imaging with a signal-to-noise ratio greater than 7 at $-30^{\circ} \mathrm{C}$ and ultralow illuminance of $10^{-4}$ lux. The detector array design will enable large focal planes, capable of imaging with high sensitivity and high speed, over a wide range of natural illumination conditions from direct sunlight to a cloudy moonless night. () 2010 SPIE and IS\&T. [DOI: 10.1117/1.3316499]
\end{abstract}

\section{Introduction}

There is a growing need in science and industry for largescale detector arrays capable of imaging with high sensitivity and high speed near room temperature, over a wide range of natural illumination conditions from direct sunlight to a cloudy moonless night. Existing solid-state camera technology does not enable imaging in the visible spectrum over such a wide dynamic range near room temperature. In this design study, we demonstrate through detailed calculation means that a novel back-illuminated, wide dynamic range avalanche photodiode (APD) device design will enable imaging with high sensitivity near room temperature at $-30{ }^{\circ} \mathrm{C}$, over the full range of natural illumination conditions from the AM 0 in space orbit around Earth, to ultralow light levels when the illuminance at the camera lens $E_{1}=0.0001$ lux [lumens $/ \mathrm{m}^{2}$ ], as on a cloudy moonless night when far from city lights. The high pixel sensitivity is achieved using a compact readout circuit, ca-

Paper 09095SSRR received May 31, 2009; revised manuscript received Sep. 28, 2009; accepted for publication Oct. 13, 2009; published online Apr. 5, 2010.

1017-9909/2010/19(2)/021102/14/\$25.00 @ 2010 SPIE and IS\&T. pable of controlling detector operation in linear and Geigermode regimes. The proposed wide dynamic range APD detector can operate in full depletion mode in the linear regime, when biased $8 \mathrm{~V}$ below the $36 \mathrm{~V}$ reverse breakdown voltage to support high quantum efficiency and speed. When charged to $44 \mathrm{~V}$ reverse bias, the detector design was shown in Ref. 1 to support Geiger-mode, and the efficiency of collecting photogenerated electrons to produce detection events was evaluated by Monte Carlo simulation to be above $80 \%$ for wavelengths between 300 and $800 \mathrm{~nm}^{1}$

The silicon APD array was designed with square pixels of $27 \mu \mathrm{m}$ side length for high resolution imaging with $100 \%$ sensitive-area-fill-factor, on a mechanically rugged and optically transparent sapphire substrate. When (100) silicon is properly etched with potassium hydroxide:isopropyl alcohol:water $\left(\mathrm{KOH}: \mathrm{IPA}: \mathrm{H}_{2} \mathrm{O}\right)$ or tetramethyl ammonium hydroxide (TMAH) solution through a thermally grown oxide mask, square-based pyramidal frustum or mesa arrays result with the four mesa sidewalls of the APD formed by (111) silicon planes that intersect the (100) planes at a crystallographic angle $\phi_{c}=54.7 \mathrm{deg}$. The APD would be fabricated in the mesa using conventional silicon processing technology. The proposed APD design enables operation over a wide dynamic range in linear mode with or without internal gain for passive imaging in daylight conditions, and in single-photon sensitive Geiger-mode for active or passive imaging under low-light level conditions. ${ }^{1,2}$ The detector is back-illuminated through a light focusing spherical microlens fabricated in the thinned sapphire substrate that directs light toward the device interior and away from the silicon mesa sidewalls, thereby compensating the optical dead space between pixels resulting from the mesa isolation etch and also from the internal device guard diode geometry.

The sapphire microlenses can be coated with an antireflective $\lambda / 4-\mathrm{MgF}_{2}$ layer, for example, to improve the optical power transmittance from air into the sapphire. In addi- 


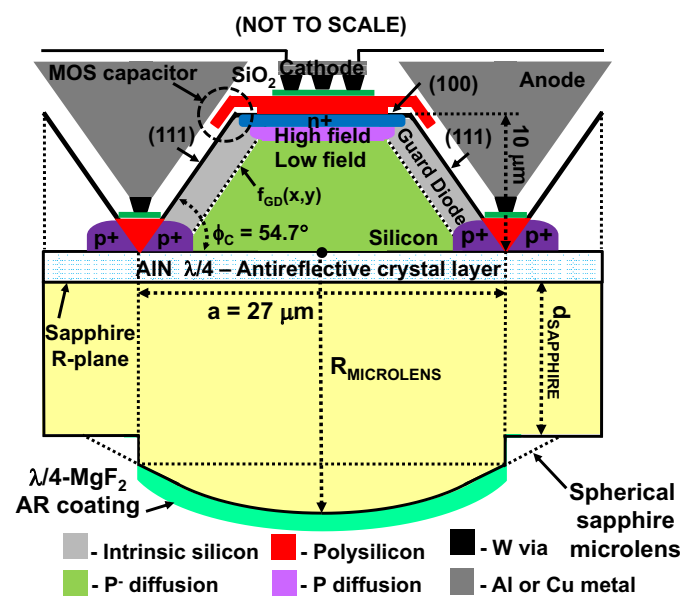

Fig. 1 Advanced back-illuminated, crystallographically etched silicon-on-( $R$-plane) sapphire APD device for arrays showing a monolithically integrated sapphire microlens and $\lambda / 4-\mathrm{MgF}_{2}$ and AIN antireflective layers.

tion, a lattice-matched (AlN) antireflective crystal layer can be grown at the sapphire-silicon interface to further increase the optical power transmittance into the device silicon, as shown in Fig. 1 and described in Ref. 2. The APDs in the array share a common, front-side anode contact, made locally at the base of each device mesa. A low resistance aluminum $(\mathrm{Al})$ or copper $(\mathrm{Cu})$ metal anode grid fills the space between pixels and also functions to inhibit optical crosstalk by shielding adjacent detectors from stray photons generated during the avalanche process. ${ }^{3}$ SOSAPD arrays are indium bump-bonded to complementary metal oxide semiconductor (CMOS) readout ICs to produce hybrid focal plane arrays (FPAs), as shown in Fig. 2, which might be $1024 \times 1024$ pixels or larger. ${ }^{4}$ The camera lens focal length and focal ratio maintain $100 \%$ sensitive-areafill-factor over the full FPA active area by limiting the maximum incidence angle of the extreme rays shown in red

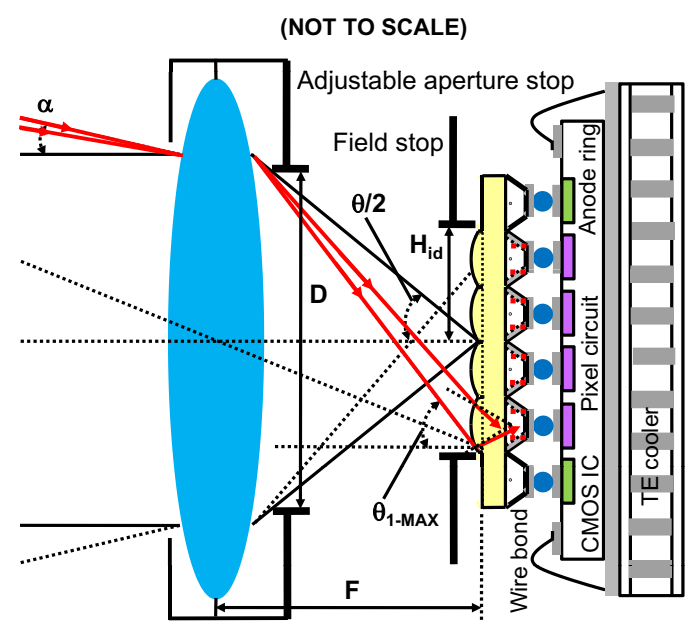

Fig. 2 Back-illuminated, silicon-on-sapphire APD array chip, bumpbonded to CMOS readout IC. Extreme rays (shown in red) are incident at the maximum incidence angle $\theta_{1-\max }$ (Color online only.) in Fig. 2, incident at the microlens edges of the pixel, located farthest from the camera optic axis at the corner of the FPA. ${ }^{2}$

The silicon APD is designed with a carefully optimized high electric field multiplication region, fabricated by high temperature diffusion of boron, described in Ref. 1. The boron impurity profile determines the spatial electric field in the device, and the boundary between the low electric field absorption-collection region and the high electric field multiplication region, as well as the APD guard diode geometry described by the guard diode boundary function $f_{\mathrm{GD}}(x, y)$, are shown in Fig. 1. The narrow, high electric field multiplication region and the much wider low electric field collection-absorption region, help to provide stable, uniform gain with minimum variance, for nearly all of the photogenerated electrons in the device. Since most electron-hole pairs (EHPs) are generated through light absorption outside of the multiplication region, this results in a low excess noise factor for the APD. ${ }^{5-7}$

\section{Device Characteristics of Wide Dynamic Range Avalanche Photodiode}

The silicon-on-sapphire material system was originally developed for integrated circuit applications; however, it also has many ideal attributes for use as a substrate, enabling back-illuminated, solid-state, silicon detector arrays. Sapphire is an anisotropic, dielectric crystal of the negative uniaxial type that is weakly birefringent $\left(n_{o}-n_{e}=0.008\right)$ and possesses broadband optical transmittance ranging from the deep ultraviolet $\left(\lambda_{o}=200 \mathrm{~nm}\right)$ to the midwave IR $\left(\lambda_{o}=5500 \mathrm{~nm}\right)$. Sapphire is extremely resilient, supporting thinning below $100 \mu \mathrm{m}$, which is an important requirement for high resolution back-illuminated detector arrays. Sapphire can be optically polished to better than an 80 to 50 (scratch-dig) surface finish and can be etched optically using $157 \mathrm{~nm}$ excimer laser ultraviolet light to fabricate light focusing microlenses for the silicon detectors. Sapphire is chemically resistant to most liquid etchants at room temperature, and therefore functions as an ideal etchstop material during liquid crystallographic etching with (KOH:IPA: $\mathrm{H}_{2} \mathrm{O}$ ) or TMAH solution to define the silicon pixel mesa arrays. To enable high quantum efficiency backilluminated silicon detector arrays, the refractive index mismatch between air, sapphire, and silicon has to be corrected. The wide bandgap semiconductor material aluminum nitride (AIN) is closely lattice matched and refractive index matched to both sapphire and silicon, and offers the prospect of enabling fabrication of high transmittance (100) silicon-on-(AIN)-sapphire substrates for backilluminated silicon imagers. ${ }^{8-10}$

The APD in Fig. 1 has been designed for wide dynamic range operation by allowing the polysilicon cathode to extend beyond the edges of the mesa top over the thermally grown passivation oxide to form an MOS capacitor that acts as a charge-coupled device (CCD) gate. When the APD is reverse biased, the combination of the APD $p / n$-junction and the MOS capacitor formed by the overhanging polysilicon operates similarly to the input diode and input gate of a CCD. ${ }^{11}$ When the wide dynamic range APD in Fig. 1 is reverse biased at voltages well below the reverse breakdown voltage of the detector, the $p / n$-junction 
cannot deplete fully to the silicon-sapphire interface due to blocking by the high electric field boron impurity diffusion. However, the polysilicon MOS capacitor can deplete into the $p$-type silicon fully to collect any photogenerated electrons in the device. The APD $p / n$-junction prevents an inversion layer from forming under the polysilicon CCD gate by collecting the photoelectrons under the gate, producing an unmultiplied photocurrent. Therefore, at low reverse bias voltages, the polysilicon MOS capacitor depletes deep into the silicon to collect photogenerated electrons, which are in turn collected by the cathode of the APD $p / n$-junction, bypassing the high electric field region of the APD without being multiplied. At low reverse bias, the photogenerated electrons are mainly directed toward the MOS capacitor at the periphery of the device and relatively few photogenerated electrons in the APD reach the high electric field multiplication region. Therefore, the internal gain of the device remains low, even though the electric field in the multiplication region might reach the critical value of $\mathbf{E}_{\text {critical }}=3 \times 10^{5} \mathrm{~V} / \mathrm{cm}$ in silicon required for impact ionization. The region at the periphery of the device is indicated as a guard diode, because its purpose is to collect surface generated electrons. The electrons collected at the periphery will have reduced probability of drifting to the high field region to be multiplied. When the APD is biased above the reverse breakdown voltage, a much larger fraction of photogenerated electrons are directed toward the multiplication region of the device than at lower bias voltages, resulting in larger internal gain. For both linear and Geiger-mode operation, the device silicon will be fully depleted to enable efficient collection of photogenerated electrons from the entire silicon volume. These results were confirmed through extensive device simulations using MEDICI software of mesa APD structures created using the TSUPREM 4 fabrication simulation software as described in Refs. 1 and 2.

\subsection{Calculated Photocurrent of Wide Dynamic Range Mesa Avalanche Photodiode}

The sensitivity of the wide dynamic range APD in Fig. 1 can be evaluated by considering that the silicon detector images primarily over the visible wavelength range. Manufacturers of commercial consumer cameras that detect primarily in the visible spectral range often describe the camera sensitivity by specifying the minimum illuminance condition at the entrance aperture of the camera in photometric units of lux required to capture an image with clarity. Table 1 lists illuminance values for different imaging conditions. 12

The natural illuminances $\left(E_{1}\right)$ in Table 1 represent values at the entrance aperture of an imaging system of the type shown in Fig. 2. The range of illuminance values for direct sunlight listed in Table 1, existing at the camera lens aperture, are produced by solar spectral irradiance at airmass AM 0 and other airmass conditions arising for different angles of the sun above the Earth's horizon. These spectral irradiance curves can be used to calculate the total photon flux incident on the $27 \mu \mathrm{m}$ wide dynamic range mesa APD pixel that produces a photocurrent in response to the illumination. Under AM 0 illumination, when the wide dynamic range APD camera stares directly at the sun from Earth's orbit in space, the AM 0 spectral irradiance is given
Table 1 Natural illumination conditions and the corresponding illuminance $E_{l}$ in photometric units at the entrance aperture or camera lens of the imaging system.

\begin{tabular}{cc}
\hline \hline Illuminance $\left(E_{l}\right)$ & Lux $\left[\right.$ lumens $\left./ \mathrm{m}^{2}\right]$ \\
\hline $\begin{array}{c}\text { Direct sunlight at noon, } \\
\text { very clear day }\end{array}$ & $\mathbf{5 0 , 0 0 0}$ to $\mathbf{1 3 0 , 0 0 0}$ \\
Daylight, sunny day & 15,000 \\
Daylight, cloudy & 2,000 \\
One hour before dusk & 200 \\
a Dusk & 10 \\
Late dusk & 1 \\
Clear night, full moon \\
Clear night, quarter moon
\end{tabular}

aegal definition of dusk.

by the plot in Fig. 3. ${ }^{13}$ Figure 4 shows the geometry of imaging the sun directly using the APD-FPA camera with a lens focal length $F=21 \mathrm{~cm}$ and focal ratio setting $f / \#=5.6$, required for $100 \%$ sensitive-area-fill-factor over the full $1024 \times 1024$ FPA area, as calculated in Ref. 2 .

The dashed blue curve in Fig. 3 represents the calculated spectral irradiance of the solar black body obtained from Eq. (1), which is derived from the Planck black body law.

$$
\begin{aligned}
F(\lambda) d \lambda & =\frac{P(\lambda)}{4 \pi r^{2}} d \lambda \\
& =\frac{2 \pi h c^{2}}{\lambda^{5}\left[\exp \left(h c / \lambda k_{b} T\right)-1\right]}\left(\frac{R_{\text {sun }}^{2}}{r^{2}}\right) d \lambda\left[\mathrm{W} / \mathrm{m}^{2}\right] .
\end{aligned}
$$

In Eq. (1), the average distance of the Earth from the sun is given by $r_{\text {sun }}=1.472 \times 10^{11} \mathrm{~m}$ and the radius of the sun $R_{\text {sun }}=6.96 \times 10^{8} \mathrm{~m}$. The G173-03 standard described in

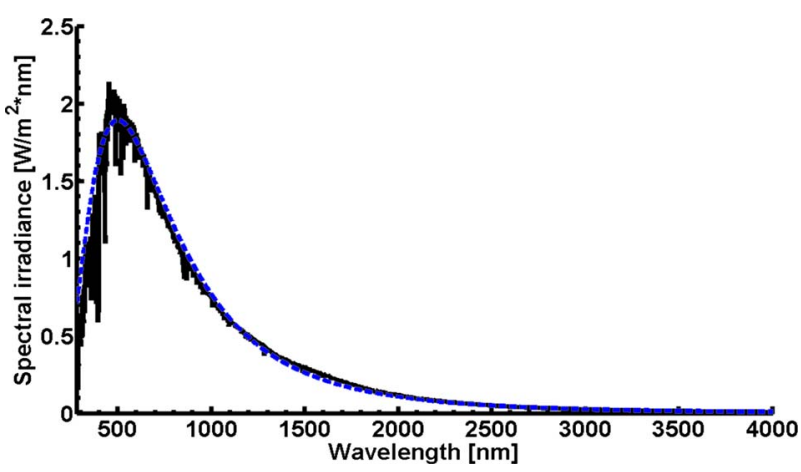

Fig. 3 Spectral irradiance of the AM 0 illumination based on the G173-03 ASTM standard (solid) and the calculated solar black body (dashed) with $T=5800 \mathrm{~K}$. 


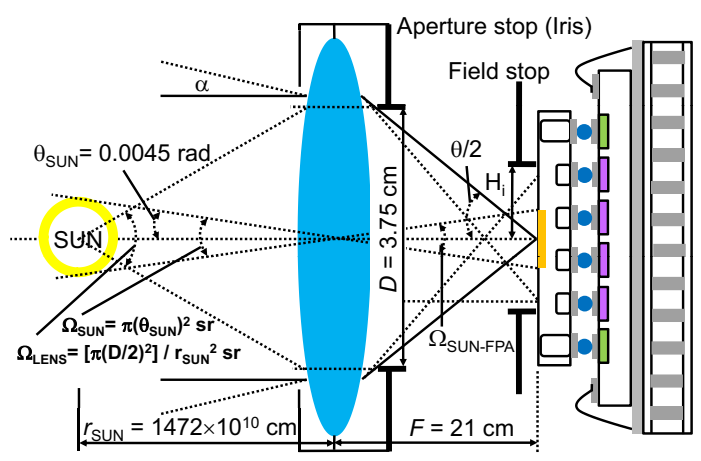

Fig. 4 Geometry of imaging the sun directly using the APD-FPA with camera focal length $F=21 \mathrm{~cm}$ and $f / \#=5.6$.

Fig. 3 is produced by the American Society for Testing and Materials (ASTM) for photovoltaic performance evaluation and also defines in addition to AM 0 (for orbit around the Earth), two standard terrestrial solar spectral irradiance curves both at AM 1.5 conditions. ${ }^{14}$

To calculate the AM 0 and AM 1.5 illuminances $E_{1}$, the radiometric AM 0 and AM 1.5 spectral irradiances need to be converted to photometric spectral illuminances $E_{1}$ [lumens $/ \mathrm{m}^{2}-\mathrm{nm}$ ] using the conversion formula in Eq. (2).

$P_{\text {lumens }}=K_{\max } \int_{0}^{\infty} V(\lambda) p(\lambda) d \lambda$.

The photopic response curve $V(\lambda)$ of the human eye is shown in Fig. 5 and Fig. 6 shows the spectral illuminance curves for solar AM 0 and AM 1.5 conditions. In Eq. (2), $K_{\max }=683$ lumens $/ \mathrm{W}$ and $p(\lambda)$ is the radiometric spectral irradiance.

Integrating the AM 0 spectral illuminance curve in Fig. 6 over wavelengths from 280 to $4000 \mathrm{~nm}$ yields a value of total illuminance that closely matches the maximum value of $E_{\text {1-AM } 0}=130,000$ lux in Table 1, as expected. Integrating the AM 1.5 Global Tilt and AM 1.5 Direct curves in Fig. 6 yields $E_{\mathrm{l}-\mathrm{AM} 1.5 \mathrm{GT}} \approx 110,000 \mathrm{lux}$ and $E_{1-\mathrm{AM} 1.5 \mathrm{D}}$ $\approx 97,000$ lux, respectively. These values represent illuminances in units of lumens $/ \mathrm{m}^{2}$ at the entrance aperture of a camera staring directly into the sun.

When imaging the sun directly, as shown in Fig. 4 using a $1024 \times 1024$ pixel focal plane array consisting of $27 \mu \mathrm{m}$,

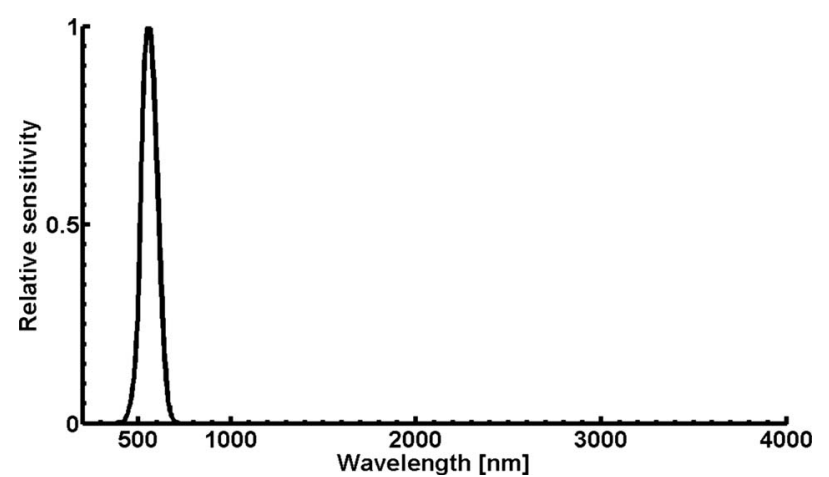

Fig. 5 Photopic response $V(\lambda)$ of the human eye. Peak response occurs at $\lambda_{o}=555 \mathrm{~nm}$.

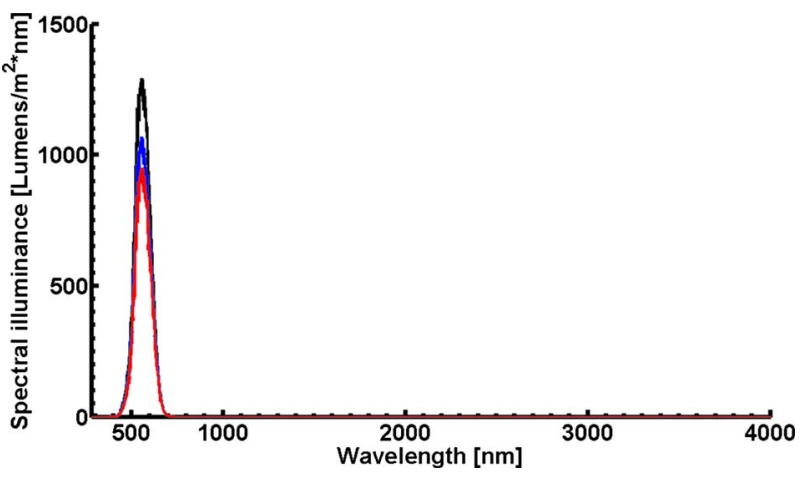

Fig. 6 Spectral illuminance curves for AM 0, AM 1.5 Global Tilt, and AM 1.5 Direct solar conditions.

wide dynamic range mesa pixels shown in Fig. 1, the photon flux incident on a pixel and the resulting photocurrent produced by the pixel can be calculated. Table 2 lists important camera parameter values for calculating the photon flux incident on the $27 \mu \mathrm{m}$ wide dynamic range APD pixel when imaging the sun directly, as shown in Fig. 4.

Using the parameter values in Table 2, the optical power $P_{\text {opt }}$ and photon flux $\Phi$ incident on a single APD pixel in the array can be calculated for different illuminance conditions at the camera lens aperture. If the camera is orbiting Earth in space and staring directly into the sun at the AM 0 condition, the irradiance at the camera lens will be $E_{\text {lens }}($ AM 0$)=1356 \mathrm{~W} / \mathrm{m}^{2}=0.1356 \mathrm{~W} / \mathrm{cm}^{2} .{ }^{13}$ The total optical power admitted by the lens is given by Eq. (3).

$P_{\text {opt-total }}=E_{\text {lens }} A_{\text {aperture }}$.

In Eq. (3), $A_{\text {aperture }}$ is the camera aperture area. According to Eq. (3), the total optical power admitted by the lens is calculated to be $P_{\text {opt-total }}=1.4916 \mathrm{~W}$. For the camera in Fig. 4,

Table 2 Parameters of the APD focal plane array camera.

\begin{tabular}{cc}
\hline \hline Parameter & Value \\
\hline Pixel size & $27 \mu \mathrm{m}$ \\
Pixel area $A_{\text {pixel }}$ & $729 \times 10^{-8} \mathrm{~cm}^{2}$ \\
Focal plane array size & $1024 \times 1024$ \\
FPA side length & $2.7648 \mathrm{~cm}^{\text {FPA area } A_{\text {FPA }}}$ \\
Camera lens focal length \\
Focal ratio setting $f / \#$ \\
Camera entrance aperture area \\
$A_{\text {aperture }}$
\end{tabular}


imaging the sun directly, the angle $\theta_{\text {sun }}=\arctan \left(R_{\text {sun }} / r_{\text {sun }}\right)$ $=0.0045 \mathrm{rad}$ and the corresponding solid angle subtended by the sun at the camera lens is given by $\Omega_{\text {sun }}$ $=\pi\left(\theta_{\text {sun }}\right)^{2}$ sr. The total optical power $P_{\text {opt-total }}=1.4916 \mathrm{~W}$, projected by the sun into $\Omega_{\text {lens }}$ under AM 0 conditions will be focused onto the 4238 pixels on the FPA that record the sun's projected image. The total optical power incident per pixel is therefore given by $P_{\text {opt-pixel }}=P_{\text {opt-total }} / 4238$ $=0.35 \mathrm{~mW}$, however, not all of this incident optical power is actually detected by the mesa APD. As the sun descends toward the horizon, higher airmass conditions apply that produce less illuminance, approaching the 50,000 lux lower limit shown in Table 1.

Imaging a scene is different from direct imaging of the sun, because the scene is subtended by the field of view of the camera and the scene is projected onto the full light sensitive area of the FPA. Using the illuminance values at the camera aperture provided in Table 1 , it is possible to calculate the total integrated photon flux and also the spectral photon flux curves with units [photons/sec-nm] as a function of wavelength that will be incident on a single $27 \mu \mathrm{m}$ mesa APD pixel shown in Fig. 1. The spectral photon flux density $\Phi(\lambda)$, incident on the $27 \mu \mathrm{m}$ mesa APD for the different scene illuminations, is used together with the previously calculated APD detector quantum efficiency $\eta(\lambda)$ from Ref. 2 to calculate the average APD photocurrent according to Eq. (4). ${ }^{15}$

$\bar{i}_{p}=\eta G q \Phi$.

The quantity $\eta$ represents the APD detector quantum efficiency and depends on the optical wavelength, while $G$ is the average internal avalanche gain of the APD, $q$ is the charge of a single electron, and $\Phi$ represents the incident photon flux. In Fig. 7, the average unmultiplied $(G=1)$ APD photocurrent $\bar{i}_{p}$ is calculated for different illumination conditions at the camera lens aperture for the $27 \mu \mathrm{m}$ mesa APD pixel in Fig. 1, without a microlens.

In Fig. 7, the APD photocurrent corresponding to a total detected photon flux of $\Phi_{\mathrm{GM}}=10^{7}$ photons $/ \mathrm{sec}$ represents the threshold photon flux for enabling Geiger-mode silicon APD operation near room temperature. ${ }^{16}$ If the mean linear mode photocurrent for the APD operating with unity gain is at or below $\left\langle i_{P-\mathrm{GM}}\right\rangle=q \Phi_{\mathrm{GM}}=1.602 \mathrm{pA}$, as shown in the figure, then Geiger-mode operation of the APD will be supported. This statement is based on the assumption that all photogenerated electrons would be multiplied in the APD to produce detection events or photon counts and also that dark count rates would be much lower than the photogenerated counts by three or more orders of magnitude to not saturate the count rate limit of the APD. The calculation in Fig. 7 shows that Geiger-mode operation of the $27 \mu \mathrm{m}$ mesa APD is possible only at night after late dusk.

The signal detected by the APD is calculated from the mean photocurrent in Fig. 7, according to Eq. (5).

$S=\left\langle i_{p}\right\rangle^{2}$

To image with high sensitivity over the normal range of illumination conditions shown in Fig. 7, the APD optical receiver electronics should be capable of supporting linear and Geiger-mode operation.

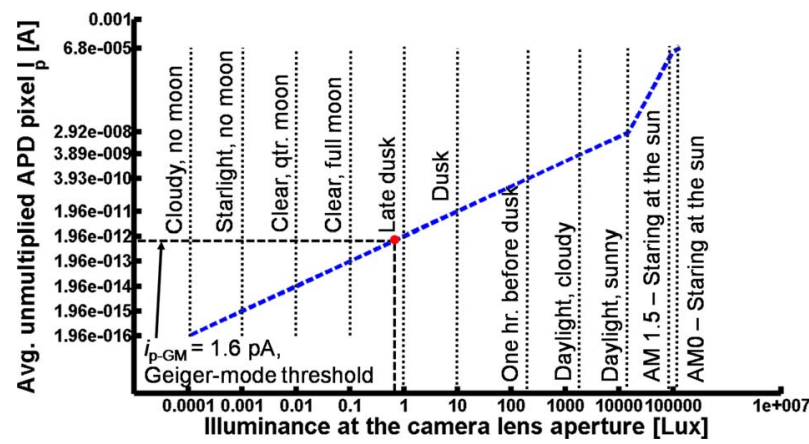

Fig. 7 APD photocurrent as a function of the illuminance at the camera lens aperture for a $27 \mu \mathrm{m}$ mesa isolated pixel without a light focusing sapphire microlens, operating at unity internal gain $G=1$. Camera lens focal length $F=21 \mathrm{~cm}$ with $f / \#=5.6$.

\subsection{Calculated Photocurrent Noise and Dark Current Noise of the Wide Dynamic Range Mesa Avalanche Photodiode}

The photoelectron and dark current noise are contributed by the mesa APD detector to the overall noise of the pixel or optical receiver, that also includes a contribution from the electronic readout noise of the pixel control circuit. The photoelectron noise can be calculated for the two terminal mesa APD with guard diode shown in Fig. 1, with parameters $\beta$ and $\gamma$ describing the probability of a photogenerated electron being collected in the active part of the device and multiplied or collected by the guard diode and not multiplied, respectively. The parameters $\beta$ and $\gamma$ are therefore random variables. The instantaneous mesa APD photocurrent with a guard diode structure is given by Eq. (6).

$$
\begin{aligned}
i_{p}(t) & =(1-R)\{1-\exp [-\alpha(\lambda) h(x, y)]\}\left(\beta+\frac{\gamma}{G}\right) G q \Phi \\
& =\frac{q G \beta}{T_{\mathrm{int}}} m+\frac{q \gamma}{T_{\mathrm{int}}} m .
\end{aligned}
$$

Equation (6) expresses the instantaneous APD photocurrent where $\beta, \gamma$, and $G$ are all random variables with assumed $\beta+\gamma=1$. The quantity $R$ represents the optical power reflectance, $\alpha(\lambda)$ is the absorption coefficient of silicon, and $h(x, y)$ is the silicon mesa height. The quantity $m$ represents the number of photogenerated electrons produced during integration time interval $T_{\text {int }}$ and $G$ describes the avalanche gain. The variables $\beta$ and $\gamma$ represent the fractions of $m$ that undergo gain multiplication or not, respectively. The mean photocurrent and the variance of the photocurrent for the mesa APD with a guard diode can be calculated. The instantaneous photocurrent in the APD with guard diode is composed of multiplied and unmultiplied photoelectrons and both are expressed by Eqs. (7) and (8), respectively. ${ }^{15}$

$i_{a}(t)=\sum_{l} X_{l} G_{l} \beta h(t-l \Delta t)$, 
$i_{g}(t)=\sum_{l} X_{l} G_{l} \frac{\gamma}{G_{l}} h(t-l \Delta t)=\sum_{l} X_{l} \gamma h(t-l \Delta t)$.

The total instantaneous photocurrent $i_{p}(t)$ can be written as the sum of Eqs. (7) and (8) as given by Eq. (9).

$i_{p}(t)=\sum_{l} X_{l} G_{l} \beta h(t-l \Delta t)+\sum_{l} X_{l} \gamma h(t-l \Delta t)$.

The mean value of the random variable $X_{l}$ is given by Eq. (10).

$\left\langle X_{l}\right\rangle=p=T \eta_{\mathrm{abs}} \Phi \Delta t$

In Eq. (10), $T$ represents the optical power transmittance into the device and $\eta_{\text {abs }}$ represents the absorption efficiency of light in the silicon. The expression for the mean photocurrent can therefore be written as shown in Eq. (11).

$$
\begin{aligned}
\left\langle i_{p}(t)\right\rangle & =\sum_{l}\left\langle X_{l}\right\rangle\left\langle G_{l}\right\rangle\langle\beta\rangle h(t-l \Delta t)+\sum_{l}\left\langle X_{l}\right\rangle\langle\gamma\rangle h(t-l \Delta t) \\
& =p\left\langle G_{l}\right\rangle\langle\beta\rangle \sum_{l} h(t-l \Delta t)+p\langle\gamma\rangle \sum_{l} h(t-l \Delta t) .
\end{aligned}
$$

Equation (11) can be solved in the limit of $\Delta t \rightarrow 0$ to yield the mean photocurrent in Eq. (12).

$$
\begin{aligned}
\left\langle i_{p}(t)\right\rangle & =T \eta_{\mathrm{abs}} \Phi\left\langle G_{l}\right\rangle\langle\beta\rangle \int_{0}^{\infty} h(t) d t+T \eta_{\mathrm{abs}} \Phi\langle\gamma\rangle \int_{0}^{\infty} h(t) d t \\
& =T \eta_{\mathrm{abs}}\left(\left\langle G_{l}\right\rangle\langle\beta\rangle+\langle\gamma\rangle\right) \Phi q .
\end{aligned}
$$

To calculate the variance of the photocurrent in the presence of random gain $G$, random $\beta$, and $\gamma$, the mean square value of the photocurrent must be calculated first according to Eq. (13) where the two currents are uncorrelated.

$\left\langle i_{p}^{2}(t)\right\rangle=\left\langle i_{a}^{2}(t)+i_{g}^{2}(t)\right\rangle$.

Calculating the mean square value of the photocurrent yields the result in Eq. (14).

$$
\begin{aligned}
\left\langle i_{p}^{2}(t)\right\rangle= & T^{2} \eta_{\mathrm{abs}}^{2} \Phi^{2}\langle G\rangle^{2}\langle\beta\rangle^{2} q^{2}+T \eta_{\mathrm{abs}} \Phi\left\langle G^{2}\right\rangle\left\langle\beta^{2}\right\rangle\left(2 q^{2} B\right) \\
& +T^{2} \eta_{\mathrm{abs}}^{2} \Phi^{2}\langle\gamma\rangle^{2} q^{2}+T \eta_{\mathrm{abs}} \Phi\left\langle\gamma^{2}\right\rangle\left(2 q^{2} B\right) \\
& +T^{2} \eta_{\mathrm{abs}}^{2} \Phi^{2}\langle G\rangle\langle\beta\rangle\langle\gamma\rangle q^{2}+T \eta_{\mathrm{abs}} \Phi\langle G\rangle\langle\beta \gamma\rangle\left(2 q^{2} B\right) .
\end{aligned}
$$

The variance of the photocurrent in the APD device with a guard diode can now be calculated according to Eq. (15) using Eqs. (12) and (14).

$$
\begin{aligned}
\sigma_{i_{p}}^{2}= & \left\langle i_{p}^{2}(t)\right\rangle-\left\langle i_{p}(t)\right\rangle^{2}=2 T \eta_{\mathrm{abs}} \Phi q^{2} B\left(F_{G}\langle G\rangle^{2} F_{\beta}\langle\beta\rangle^{2}\right. \\
& \left.+F_{\gamma}\langle\gamma\rangle^{2}+F_{a g}\langle G\rangle\langle\beta\rangle\langle\gamma\rangle\right)-T^{2} \eta_{\mathrm{abs}}^{2} q^{2} \Phi^{2}\langle G\rangle\langle\beta\rangle\langle\gamma\rangle .
\end{aligned}
$$

Since $G, \beta$, and $\gamma$ are random variables, a relationship between their mean square values and the square of the means can be defined using excess noise factors as formulated in Eq. (15) ${ }^{6,17}$ The excess noise factors for $G, \beta, \gamma$, and $\beta \gamma$ are provided in Eq. (16).

$$
F_{G}=\frac{\left\langle G^{2}\right\rangle}{\langle G\rangle^{2}} ; \quad F_{\beta}=\frac{\left\langle\beta^{2}\right\rangle}{\langle\beta\rangle^{2}} ; \quad F_{\gamma}=\frac{\left\langle\gamma^{2}\right\rangle}{\langle\gamma\rangle^{2}} ; \quad F_{a g}=\frac{\langle\beta \gamma\rangle}{\langle\beta\rangle\langle\gamma\rangle} .
$$

Equation (15) describes the photoelectron noise for the mesa APD structure shown in Fig. 1, with two terminals, a guard diode region and random gain. In Eq. (15), the mean values for $\beta$ and $\gamma$ are calculated as a function of the optical wavelength for a particular guard diode boundary function $f_{G D}(x, y)$, seen in Fig. 1 . The excess noise factors associated with $\beta, \quad \gamma$, and $\beta \gamma$ can be determined experimentally in a fabricated device or by Monte Carlo simulation. ${ }^{18}$

In addition to the photoelectron noise contribution by the mesa APD device, the thermally generated dark current in the detector also contributes to the overall noise. The contribution to the detector dark current in the mesa APD structure of Fig. 1 with a guard diode will be composed of multiplied and unmultiplied dark current. The thermally generated dark current $i_{D}$ in the mesa APD device can be defined and measured under low reverse bias conditions with the APD $p / n$-junction fully depleted to the silicon(AIN)-sapphire interface, with a peak electric field below that needed for avalanche gain in the device. ${ }^{19}$ According to this definition, the mean APD dark current is given by Eq. (17).

$\bar{i}_{D}=q \Phi_{e}=\frac{q}{T_{\mathrm{int}}} \bar{m}_{D}$

In Eq. (17), the quantity $\Phi_{e}$ describes the average number of thermally generated electrons per second and $q$ is the charge of an electron. The quantity $\bar{m}_{D}$ describes the average number of dark electrons generated during integration time interval $T_{\text {int }}$. The thermally generated mean dark current $\left\langle i_{D}\right\rangle$ can be calculated to yield an estimate for the reverse biased silicon mesa APD $p / n$-junction. Assuming the dark current is only generated thermally in the silicon bulk, $p<n_{i}$ and $n<n_{i}$, where $n_{i}$ is the silicon intrinsic carrier concentration and only a single type of trap with an energy located inside the bandgap participates; then the recombination rate $U$ is described by Eq. (18). ${ }^{20}$

$$
U=-n_{i}\left[\frac{\sigma_{p} \sigma_{n} v_{T H} N_{T}}{\sigma_{n} \exp \left(\frac{E_{T}-E_{i}}{k T}\right)+\sigma_{p} \exp \left(\frac{E_{i}-E_{T}}{k T}\right)}\right] .
$$

In Eq. (18), the quantities $\sigma_{n}$ and $\sigma_{p}$ represent the electron and hole capture cross section probabilities, $v_{T H}$ is the carrier thermal velocity, $N_{T}$ is the trap density, $E_{T}$ is the trap energy level, $E_{i}$ is the intrinsic Fermi level, and $n_{i}$ is the intrinsic carrier density. Many of the quantities in Eq. (18) depend on temperature as does the recombination rate itself. The thermally generated dark current density from the silicon bulk is calculated by integrating Eq. (18) over the full width $W$ of the reverse biased mesa APD $p / n$-junction depletion region, as given by Eq. (19).

$J_{\mathrm{gen}}=\int_{0}^{W} q|U| d x=q|U| W=\frac{q n_{i} W}{\tau_{n}}$.

In Eq. (19), the quantity $\tau_{n}$ represents the mean electron life time in $p$-type silicon material. An estimate of the mean 
APD dark current from Eq. (19) is obtained by multiplying the result by the cross sectional area $A_{\mathrm{APD}}$ of the mesa APD device to yield Eq. (20).

$\left\langle i_{D \text {-estimated }}\right\rangle=\frac{q n_{i} A_{\mathrm{APD}} W}{\tau_{n}} ; \quad \tau_{n}=\frac{1}{\sigma_{n} v_{T H} N_{T}}$.

The quantity $\tau_{n}$ in Eq. (20), depends on the electron capture cross section, carrier thermal velocity, and on the trap concentration. High values for $\tau_{n}$ in a silicon $p / n$-junction can reach $100 \mu \mathrm{sec}^{21,22}$ Equation (20) assumes that the dark current arises from the silicon bulk and that surface generated dark current contribution to the total dark current is zero. This approximation is valid for well passivated mesa sidewalls. The formalism presented here describes the probabilities for dark electrons to be collected by the active mesa APD versus the guard diode and allows sufficient flexibility for the inclusion of the surface generated dark current contribution if needed.

When the reverse bias voltage on the APD is increased to the level of avalanche gain onset, a fraction of $\Phi_{e}$ will be multiplied and the instantaneous dark current in the APD will be described by the sum of multiplied dark electrons collected in the active silicon and unmultiplied dark electrons collected in the guard diode as given by Eq. (21).

$i_{D}(t)=i_{D a}(t)+i_{D g}(t)$,

$i_{D a}(t)=\sum_{l} X_{l} \eta_{a l} G_{l} h(t-l \Delta t)$,

$i_{D g}(t)=\sum_{l} X_{l} \eta_{g l} h(t-l \Delta t)$

In Eq. (21), the quantities $\eta_{a l}, \eta_{g l}$, and $G_{l}$ are random variables with random values for each collection event. The quantity $\eta_{a l}$ describes the probability of a thermally generated electron being collected in the active region and therefore being multiplied in the APD, while $\eta_{g l}$ describes the probability of a thermally generated electron being collected by the guard diode and therefore not being multiplied. The values $G_{l}$ represent random variables of the gain imparted to a thermal electron generated during the time interval $l$.

The random variable $X_{l}$ is described by Eq. (22).

$X_{l}=\left\{\begin{array}{cc}0 \rightarrow & 1-p \\ 1 \rightarrow & p\end{array}\right\}$

The probability $p=\Phi_{e} \Delta t$ represents the probability of generation of a thermal electron during the time interval $\Delta t$. It is assumed as before that the variables $\left\{X_{l}\right\}$ are independent and the variables $\left\{G_{l}\right\}$ are also independent. The mean dark current can now be expressed using Eqs. (21) and (22) according to Eq. (23).

$$
\begin{aligned}
\left\langle i_{D}\right\rangle & =\sum_{l}\left\langle X_{l}\right\rangle\left\langle\eta_{a l}\right\rangle\left\langle G_{l}\right\rangle h(t-l \Delta t)+\sum_{l}\left\langle X_{l}\right\rangle\left\langle\eta_{g l}\right\rangle h(t-l \Delta t) \\
& =\Phi_{e}\left\langle\eta_{a l}\right\rangle\left\langle G_{l}\right\rangle \sum_{l} h(t-l \Delta t) \Delta t+\Phi_{e}\left\langle\eta_{g l}\right\rangle \sum_{l} h(t-l \Delta t) \Delta t .
\end{aligned}
$$

Taking the limit $\Delta t \rightarrow 0$, the sums in Eq. (23) become integrals according to Eq. (24).

$\lim _{\Delta t \rightarrow 0} \sum_{l} h(t-l \Delta t)=\int_{0}^{\infty} h(t) d t=q$.

The mean dark current can now be expressed according to Eq. (25).

$$
\begin{aligned}
\left\langle i_{D}\right\rangle & =\Phi_{e}\left\langle\eta_{a l}\right\rangle\left\langle G_{l}\right\rangle \int_{0}^{\infty} h(t) d t+\Phi_{e}\left\langle\eta_{g l}\right\rangle \int_{0}^{\infty} h(t) d t \\
& =\Phi_{e}\left\langle\eta_{a l}\right\rangle\left\langle G_{l}\right\rangle q+\Phi_{e}\left\langle\eta_{g l}\right\rangle q \\
& =\Phi_{e} q\left(\left\langle\eta_{a l}\right\rangle\left\langle G_{l}\right\rangle+\left\langle\eta_{g l}\right\rangle\right) .
\end{aligned}
$$

To calculate the dark current variance, the mean square dark current is calculated to yield the result in Eq. (26).

$$
\begin{aligned}
\left\langle i_{D}^{2}(t)\right\rangle= & \Phi_{e}^{2}\langle G\rangle^{2}\left\langle\eta_{a}\right\rangle^{2} q^{2}+\Phi_{e}\left\langle G^{2}\right\rangle\left\langle\eta_{a}^{2}\right\rangle\left(2 B q^{2}\right)+\Phi_{e}^{2}\left\langle\eta_{g}\right\rangle^{2} q^{2} \\
& +\Phi_{e}\left\langle\eta_{g}^{2}\right\rangle\left(2 B q^{2}\right)+\Phi_{e}^{2}\langle G\rangle\left\langle\eta_{a}\right\rangle\left\langle\eta_{g}\right\rangle q^{2} \\
& +\Phi_{e}\langle G\rangle\left\langle\eta_{a} \eta_{g}\right\rangle\left(2 B q^{2}\right)
\end{aligned}
$$

The variance of the dark current is given using Eqs. (25) and (26) according to Eq. (27).

$$
\begin{aligned}
\sigma_{i_{D}}^{2}= & 2 \Phi_{e} q^{2} B\left(F_{G} F_{a}\langle G\rangle^{2}\left\langle\eta_{a}\right\rangle^{2}+F_{g}\left\langle\eta_{g}\right\rangle^{2}\right. \\
& \left.+F_{a g}\langle G\rangle\left\langle\eta_{a}\right\rangle\left\langle\eta_{g}\right\rangle\right)-\Phi_{e}^{2} q^{2}\left\langle\eta_{a}\right\rangle\left\langle\eta_{g}\right\rangle\langle G\rangle .
\end{aligned}
$$

Since $\eta_{a}$ and $\eta_{g}$ are random variables, a relationship between their mean square values and the square of the means is defined using excess noise factors. The excess noise factors for $G, \eta_{a}, \eta_{g}$, and $\eta_{a} \eta_{g}$ are given in Eq. (28).

$F_{G}=\frac{\left\langle G^{2}\right\rangle}{\langle G\rangle^{2}} ; \quad F_{a}=\frac{\left\langle\eta_{a}^{2}\right\rangle}{\left\langle\eta_{a}\right\rangle^{2}} ; \quad F_{g}=\frac{\left\langle\eta_{g}^{2}\right\rangle}{\left\langle\eta_{g}\right\rangle^{2}} ; \quad F_{a g}=\frac{\left\langle\eta_{a} \eta_{g}\right\rangle}{\left\langle\eta_{a}\right\rangle\left\langle\eta_{g}\right\rangle}$.

Equation (27) describes the dark current noise for the mesa APD pixel design in Fig. 1 with two terminals, a guard diode region and random gain. In Eq. (27), estimates for the mean values of $\eta_{a}$ and $\eta_{g}$ can be made by assuming that $\Phi_{e}$ has a contribution only from the thermally generated bulk dark current. The thermally generated electron flux $\Phi_{e}$ is calculated from Eqs. (20) and (17) and depends on the temperature. For the case of zero surface dark current, $\left\langle\eta_{a}\right\rangle$ represents the fractional volume of active silicon to the total device mesa silicon volume and $\left\langle\eta_{g}\right\rangle$ represents the fractional volume of guard diode silicon to the total mesa silicon volume. The pyramidal mesa frustum volume is given by Eq. (29). 


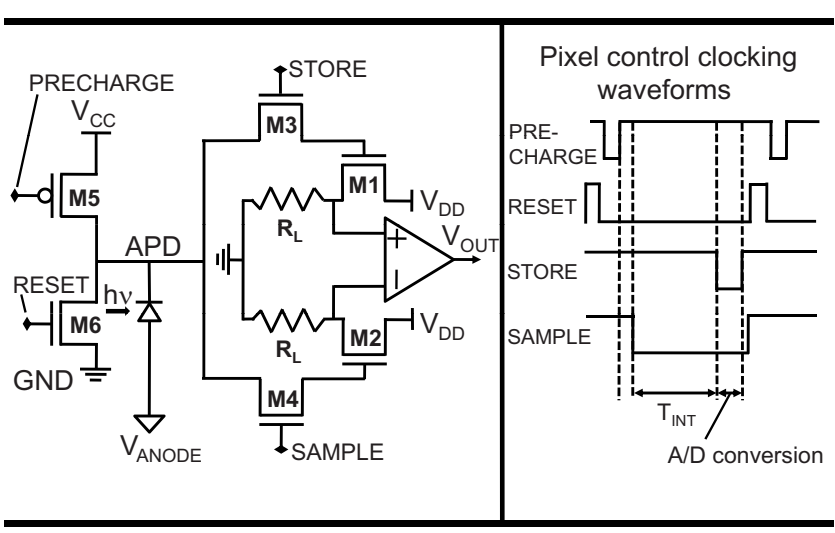

Fig. 8 Analog readout circuit controls APD in the linear mode using the precharge method.

$V_{\text {frustum }}=\frac{1}{3} h\left(A_{1}+A_{2}+\sqrt{A_{1} A_{2}}\right)$

In Eq. (29), $A_{1}$ is the pyramidal frustum base area and $A_{2}$ is the pyramidal frustum top area. For the $27 \mu \mathrm{m}$ pixel mesa of height $h=10 \mu \mathrm{m}$, with a guard diode boundary defined by parameter $i_{G D}=1 \mu \mathrm{m}$ with the same slope as the mesa sidewall as shown in Fig. 1, the mean fractional volume of the active and guard silicon is given by Eq. (30).

$\left\langle\eta_{a}\right\rangle=\frac{V_{\text {active }}}{V_{\text {frustum }}}=0.817$,

$\left\langle\eta_{g}\right\rangle=\frac{V_{\text {guard }}}{V_{\text {frustum }}}=0.183$.

It is assumed for the calculation of the $27 \mu \mathrm{m}$ mesa APD pixel that the boundaries between the guard diode region and active region in the device are defined by the fixed boundary in Fig. 1, for which case the excess noise factors $F_{a}$ and $F_{g}$ in Eq. (28) are reduced to unity.

\section{Pixel Circuit Design}

The pixel sensitivity depends on the electronic readout circuit design which must be capable of controlling linear and Geiger-mode operation of the detector as shown in Fig. 7, to image successfully over the full range of natural illumination conditions. The pixel readout circuit in Fig. 8, based on a voltage source follower amplifier, may be used to control linear mode operation of the mesa APD and the circuit in Fig. 9 to control Geiger-mode operation.

The linear mode electronic readout circuit for the APD in Fig. 8 combines a voltage source follower amplifier consisting of transistor M1 and load resistor $R_{L}$, with pass transistor M3 to store the charge integrated at the gate capacitance of M1. The circuit is shown in the figure to be capable of performing correlated double sampling (CDS) using a second matching voltage source follower amplifier made up of transistor M2 with its own identical load resistor $R_{L}$ and pass transistor M4. The readout circuit in Fig. 9 implements active quenching for the Geiger-mode APD to shorten the detector reset time. During a normal image acquisition cycle, the APD and readout circuit are first reset

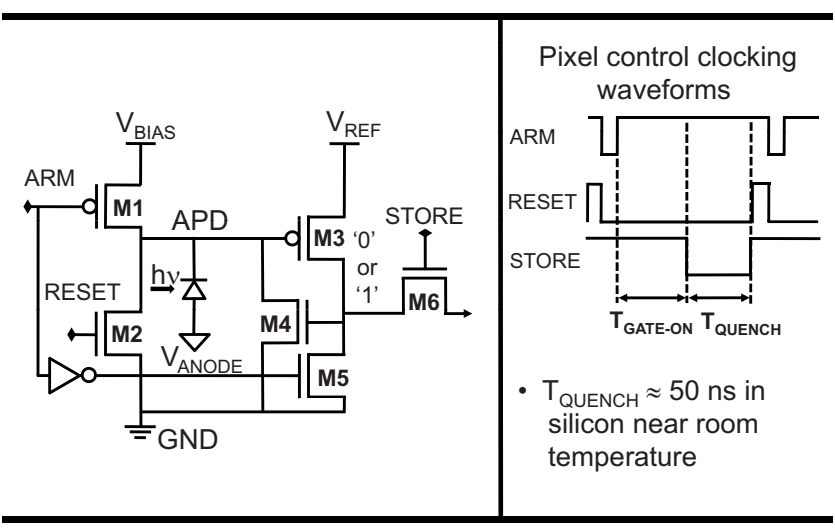

Fig. 9 Digital readout circuit controls APD in the single photon sensitive Geiger-mode using active quenching.

by turning on then off reset transistor M2. Transistor M1 is then used to precharge the APD and the gate capacitance of M3 to the $V_{\text {bias }}$ dc supply voltage. In response to just a single detected photon, the large avalanche gain in the APD $\left(G>10^{6}\right)$ produces a photocurrent capable of quickly discharging the self-capacitance of the APD. The capacitance at the gate of M3 is also discharged by the APD photocurrent, thereby turning on M3. The transistor M3 senses the onset of the avalanche in the APD and turns on M4 which diverts a significant fraction of the charge to ground instead of it flowing through the APD junction where it can fill traps and lengthen the quench time of the detector following a detection event. An advantage of the circuits in Figs. 8 and 9 includes being able to combine them into a single compact pixel readout circuit that is capable of controlling both linear and Geiger-mode operation for the wide dynamic range mesa APD detector as shown in Fig. 10.

The circuit in Fig. 10 combines the analog APD readout circuit shown on the right with a digital readout circuit shown on the left. When the APD is biased in linear mode, transistor M7 is turned off and the APD is controlled by the analog circuit on the right. When the APD is biased in Geiger-mode, transistors M3 and M4 are turned off and M7 is turned on, allowing the APD to be controlled by the digital circuit on the left.

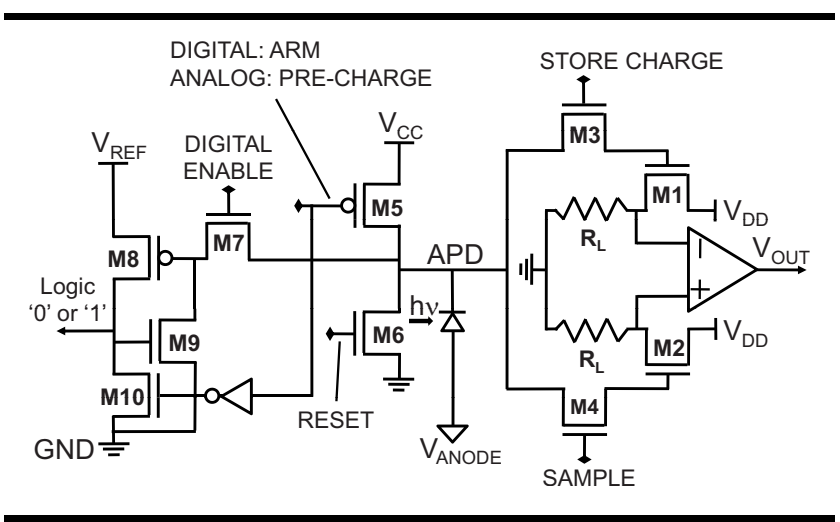

Fig. 10 APD readout circuit combines analog and digital readout circuits to enable linear and Geiger-mode APD detector operation for wide dynamic range imaging. 
Stern and Cole: High-sensitivity, wide-dynamic-range avalanche photodiode pixel design...

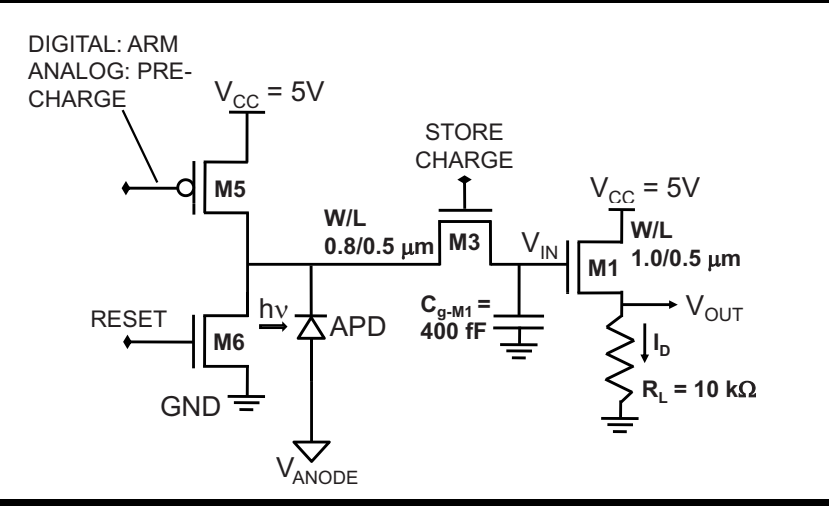

Fig. 11 APD pixel readout circuit is designed using the TSMC $0.25 \mu \mathrm{m}$ RF-CMOS process. M1 is biased into the constant current region after the linear capacitor $C_{g-\mathrm{M} 1}$ is precharged. Analog APD readout circuit is shown without CDS.

\subsection{Linear Mode Circuit Characteristics}

During a normal image frame acquisition by the linear circuit, both pass transistors M3 and M4 are initially conducting or turned on. Transistor M6 resets the APD as indicated in the pixel control clocking waveforms. Transistor M5 is used to precharge the APD self-capacitance and the gate capacitance of M1 and M2 to the $V_{C C}$ supply voltage. Transistor M4 is allowed to stay turned on briefly after M5 is turned off to allow the voltages at the gates of M1 and M2 to equalize. Following the voltage equalization, M4 is turned off, marking the start of the frame integration time $T_{\text {int }}$, indicated in Fig. 8. During the integration time a photocurrent $i_{p}$, produced by incident illumination on the APD, discharges the precharged self-capacitance of the APD as well as the integration capacitance at the gate of transistor M1. At the end of the charge integration, pass transistor M3 is turned off and the charge is stored on the integration capacitor at the gate terminal of M1 which also serves as the input to the voltage source follower amplifier. Following the image frame integration time, the analog voltage output from the pixel circuit can be digitized using an A/D converter located on the periphery of the pixel array. The linear mode control circuit in Fig. 8 can provide low noise using CDS.

In contrast to the digital readout circuit that contributes zero electronic readout noise, the analog circuit composed of the voltage source follower and pass transistor with integration capacitor at the input of the voltage source follower, contributes to the electronic noise. Correlated double sampling shown in Fig. 10 can be used to reduce the noise contribution from the integration capacitor, however, such CDS circuitry may increase the area footprint required for the dual mode readout circuit in Fig. 10, beyond the $27 \times 27 \mu \mathrm{m}$ limit. Therefore, it is more appropriate in the noise analysis to consider the readout circuit for linear mode operation without CDS. Such an analysis is also more conservative because it assumes the worst case noise contribution from the circuit. Figure 11 shows the linear mode readout circuit without $\mathrm{CDS}$ used in the signal-to-noise ratio analysis of the APD pixel.

The circuit in Fig. 11 is designed for implementation
Table 3 Linear mode electronic readout circuit parameters.

\begin{tabular}{cc}
\hline \hline Parameter & Value \\
\hline M1 gate length & $L=0.5 \mu \mathrm{m}$ \\
M1 gate width & $W=1 \mu \mathrm{m}$ \\
Threshold voltage & $V_{\mathrm{TN} 0}=0.45 \mathrm{~V}$ \\
Gate oxide thickness & $t_{O X}=8 \mathrm{~nm}$ \\
Load resistor $R_{L}$ & $R_{L}=10,000 \Omega$ \\
Integration capacitor $C_{g-\mathrm{M} 1}$ & $C_{g-\mathrm{M} 1} \equiv C_{\mathrm{int}}=401.43 \mathrm{fF}$ \\
\hline \hline
\end{tabular}

using the TSMC $0.25 \mu \mathrm{m}$ RF-CMOS process, and Table 3 lists important parameter values for the circuit.

In Table 3, the gate length of M1 is given as $L=0.5 \mu \mathrm{m}$ because the foundry process offers a $5 \mathrm{~V}$ transistor with a thicker gate oxide and longer gate length. Using the higher voltage transistor in the pixel circuit allows biasing M1 into the active region when the gate capacitance is precharged at the start of signal integration. The RFCMOS fabrication process supports a metal capacitor with capacitance per unit area of $1 \mathrm{fF} / \mu \mathrm{m}^{2}$. Such a capacitor is linear and with a total area of $20 \times 20 \mu \mathrm{m}$, provides $400 \mathrm{fF}$ of capacitance at the gate of transistor M1. The total capacitance at the gate of M1 is given by Eq. (31).

$C_{g-\mathrm{M} 1}=\frac{2}{3} W L C_{O X}+400 \mathrm{fF}=1.43 \mathrm{fF}+400 \mathrm{fF}=401.43 \mathrm{fF}$.

Transistor M1 of the voltage source follower in Fig. 11 can be biased into the active region with $V_{D S}>V_{G S}-V_{T N}$, when $C_{g-\mathrm{M} 1}$ is precharged by M5 to $V_{\mathrm{IN}}=V C C-V_{T N} \leq 4.5 \mathrm{~V}$. The total charge stored on $C_{g-\mathrm{M} 1}$ for $V_{\mathrm{IN}}=4.5 \mathrm{~V}$ will therefore be given by Eq. (32).

$$
\begin{aligned}
Q_{C_{g}-\mathrm{M} 1}= & C_{g-\mathrm{M} 1} V_{\mathrm{IN}}=1.8 \times 10^{-12} \text { coulombs } \\
& \rightarrow 1.13 \times 10^{7} \text { electrons. }
\end{aligned}
$$

The input voltage $V_{\text {IN }}$ at the gate of M1 is given by the initial charge $Q_{C_{g}-\mathrm{M} 1}$ deposited on the integration capacitance $C_{g-\mathrm{M} 1}$ corresponding to the precharge voltage $V_{C C}$, minus the charge that was sourced by the photocurrent flowing through the APD during the integration time interval given by Eq. (33).

$V_{\mathrm{IN}}=V_{C C}-\frac{\left\langle i_{p}\right\rangle T_{\mathrm{int}}}{C_{g-\mathrm{M} 1}}$.

The voltage transfer characteristic of the source follower amplifier in Fig. 11 can be calculated by knowing that M1 operates in the active region. If the integration capacitor $C_{g-\mathrm{M} 1}$ is precharged to a voltage $V_{\mathrm{IN}}=4.5 \mathrm{~V}$ as described in Eq. (32), the voltage transfer characteristic for the amplifier output voltage $V_{\text {OUT }}$ as a function of $V_{\text {IN }}$ can be calculated from Eq. (34) where $V_{T N}$ is calculated iteratively taking into consideration the body effect. 


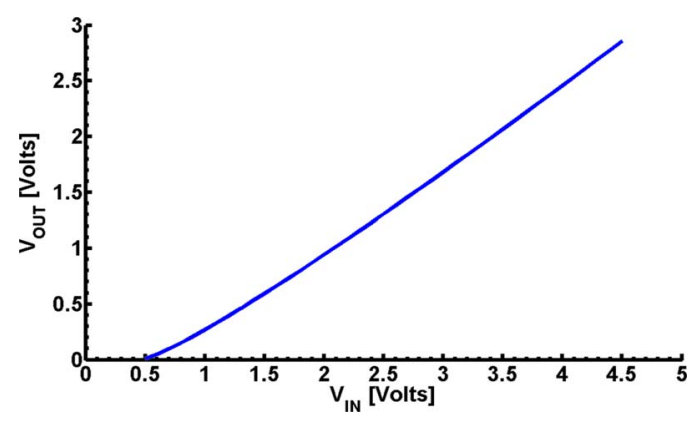

Fig. 12 Source follower voltage amplifier linear output $V_{\text {OUT }}$ as a function of input voltage $V_{\mathbb{I N}}$.

$$
\begin{aligned}
V_{\mathrm{OUT}}= & V_{\mathrm{IN}}-V_{T N}+\frac{1}{\left(\mu_{n} C_{o x} \frac{W}{L}\right) R_{L}}-\left\{\frac{2\left(V_{\mathrm{IN}}-V_{T N}\right)}{2\left[\left(\frac{1}{2} \mu_{n} C_{o x} \frac{W}{L}\right) R_{L}\right]}\right. \\
& +\left[2\left(\frac{1}{2} \mu_{n} C_{o x} \frac{W}{L}\right) R_{L}\right]^{2}
\end{aligned}
$$

The voltage transfer characteristic of the voltage source follower amplifier is plotted in Fig. 12.

\subsection{Linear Mode Circuit Noise Characteristics}

The electronic noise from the linear mode circuit in Fig. 11 is obtained by analyzing the small signal noise models.

The resistor $r_{d s 1}$ in Fig. 13 represents the finite output impedance of M1 including channel length modulation effects. The possible difference in potential between the source and body terminals of M1, gives rise to a voltagecontrolled current source with current given by $g_{s 1} V_{s 1}$. As a result, it can be modeled as a resistor with value equal to $1 / g_{s 1}$, as shown in Fig. 13. Since the three resistances $R_{L}$, $r_{d s 1}$, and $1 / g_{s 1}$ are in parallel with $R_{L}\left\|r_{d s 1}\right\| 1 / g_{s 1}$ and since $R_{L} \ll r_{d s 1}$ and also $R_{L} \ll 1 / g_{s 1}$, then the noise due to the load resistor $R_{L}$ will dominate as shown in Fig. 13. The noise from MOSFET M1 is also indicated in Fig. 13. The finite resistance of MOSFET channel M3 contributes to the noise of the sample and hold output shown in Fig. 14.

The noise voltage from the sample and hold circuit in Fig. 14 is contributed at the gate of MOSFET M1 and this can be converted into a noise current variance at the output of the source follower amplifier according to Eq. (35). ${ }^{23}$

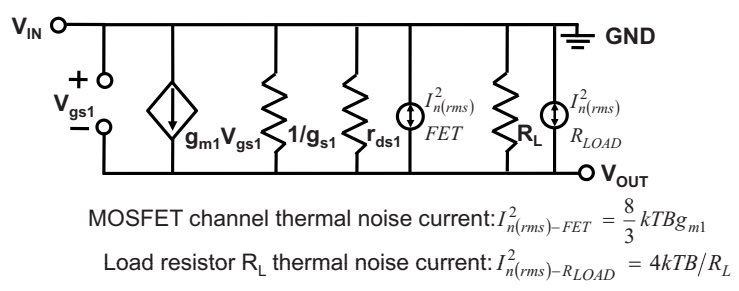

Fig. 13 Small signal noise model of the source follower amplifier of Fig. 11 showing noise current sources from a resistive MOSFET channel and from the load resistor $R_{L}$. Noise sources are in parallel and can be added to yield total output noise current.

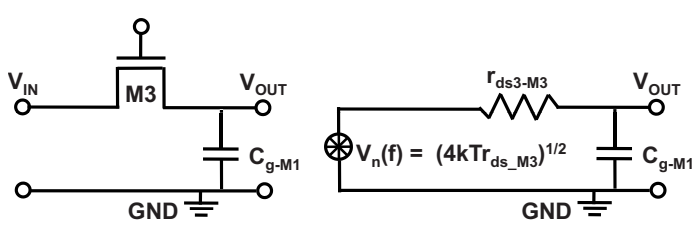

Fig. 14 Sample and hold circuit of the source follower amplifier of Fig. 11 and the noise model.

$\sigma_{i_{n \text {-sample and hold }}^{2}}=\frac{k T\left(g_{m 1}\right)^{2}}{C_{g-\mathrm{M} 1}}$.

The total noise from the linear APD readout circuit of Fig. 11 is therefore expressed according to Eq. (36).

$$
\begin{aligned}
\sigma_{i_{e n}}^{2} & =\sigma_{i_{n F E T}}^{2}+\sigma_{i_{n R}}^{2}+\sigma_{i_{n S} \text { and } \mathrm{H}}^{2} \\
& =\frac{8}{3} k T B g_{m 1}+4 k T B / R_{L}+\frac{k T\left(g_{m 1}\right)^{2}}{C_{g-\mathrm{M} 1}} .
\end{aligned}
$$

The electronic noise result from Eq. (36) is used in the sensitivity analysis of Sec. 4 for the wide dynamic range mesa APD optical receiver based on the $27 \mu \mathrm{m}$ mesa APD of Fig. 1.

\section{Sensitivity of the Linear and Geiger-Mode Avalanche Photodiode Optical Receiver}

The sensitivity or signal-to-noise of the wide dynamic range mesa APD optical receiver is calculated using the mean photocurrent given in Eq. (4), together with the variance of the photocurrent in Eq. (15), the dark current in Eq. (27) and the electronic circuit readout noise in Eq. (36) according to Eq. (37).

$\mathrm{SNR}_{\text {APD-linear-mode-receiver }}=\frac{\left\langle i_{p}(t)\right\rangle^{2}}{\sigma_{i_{p}}^{2}+\sigma_{i_{D}}^{2}+\sigma_{i_{e n}}^{2}}$.

Other sources of noise such as the background noise which is a type of photon noise associated with light from extraneous optical sources other than the signal of interest, is not considered in the analysis. Background noise is mainly a problem when imaging at midwave IR and longwave IR wavelengths where objects in a scene emit radiation according to the Planck black body law. ${ }^{17}$ Leakage currents are also not considered in the noise analysis because the surface leakage currents usually occur at poorly passivated surfaces of semiconductor $p / n$-junctions, for example, when ions on the semiconductor surface induce mirror charges in the semiconductor that form surface channels, enabling a surface leakage current to flow. ${ }^{20}$ Bulk leakage currents may occur as a result of defects in the crystal structure of the bulk semiconductor material. In the mesa structure APD design presented here, the surfaces of the mesa are passivated with thermally grown silicon dioxide on the (100) and (111) planes. Therefore, it is expected that surface leakage currents at the APD $p / n$-junction will be minimized through good quality thermally grown oxide passivation. Surface leakage currents at the (111) mesa sidewalls of the APD are expected to be negligible compared to the bulk dark current in the device. The APD guard 
Table 4 Sensitivity calculation parameters.

\begin{tabular}{cc}
\hline \hline Parameter & Value \\
\hline $\begin{array}{c}\text { APD focal plane } \\
\text { array temperature } \\
\text { APD array size }\end{array}$ & $\begin{array}{c}T=243 \mathrm{~K} \text { (two-stage } \\
\text { thermoelectric cooler) }\end{array}$ \\
APD pixel size & $1024 \times 1024$ pixels \\
APD mesa pixel height & $a=27 \mu \mathrm{m}$ \\
Microlens & $h=10 \mu \mathrm{m}$ \\
Camera lens focal length & No microlens \\
Camera focal ratio & $F=21 \mathrm{~cm}$ \\
Minority carrier lifetime $\tau_{n}$ & $f / \#=5.6$ \\
in APD silicon & $\tau_{n}=100 \mu \mathrm{sec}^{22}$ \\
APD ionization ratio & $k_{e, h}=0.2$ used to calculate \\
$F_{G}$ after Mclntyre \\
Transconductance of M1 \\
Load resistor $R_{L}$
\end{tabular}

diode at the device periphery will also collect and limit the multiplication of any surface leakage currents. ${ }^{19}$

The signal-to-noise ratio is calculated for the linear mode $27 \mu \mathrm{m}$ mesa APD pixel according to Eq. (37) for the normal range of illumination conditions with the following assumptions listed in Table 4.

Using the parameter values listed in Table 4, the signalto-noise ratio for the wide dynamic range APD optical receiver in linear mode is calculated according to Eq. (37) for different natural illumination conditions. The results are shown in Figs. 15-21 for AM 0 to late dusk.

In Fig. 15 the APD stares into the sun and unity gain provides SNR above the unity threshold. The maximum integration time $T_{\text {int-max }}=17.6 \mathrm{~ns}$ is also indicated due to the large APD photocurrent that quickly discharges the integration capacitor of the linear readout circuit.

In Fig. 16 the maximum integration $T_{\text {int-max }}=25.7 \mathrm{~ns}$ is indicated because of the large APD photocurrent that quickly discharges the integration capacitor of the linear readout circuit.

In Fig. 17, gain $>3$ becomes necessary to provide larger than unity signal-to-noise ratio.

In Fig. 18, gain $>30$ becomes necessary to provide larger than unity signal-to-noise ratio.

In Fig. 19, gain $>200$ becomes necessary to provide larger than unity signal-to-noise ratio.

In Fig. 20, gain $>5000$ becomes necessary to provide larger than unity signal-to-noise ratio.

In Fig. 21, gain $>50,000$ becomes necessary to provide larger than unity signal-to-noise ratio. At late dusk, the capability of the linear mode imager to provide high sensitiv-

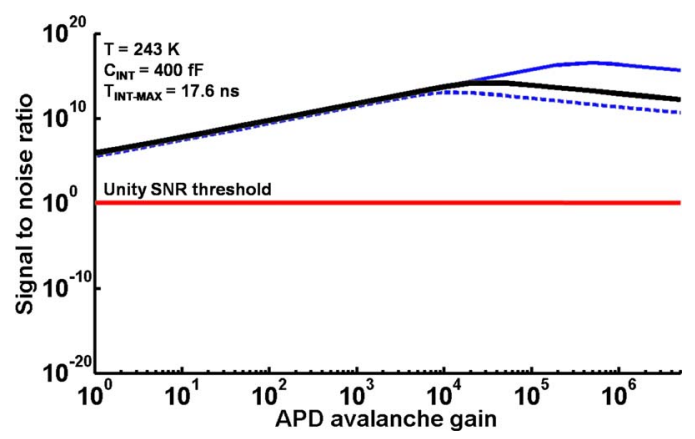

Fig. 15 SNR of the linear mode APD imager under AM 0 (130,000 lux) illumination. Bandwidths of $1 \times 10^{4} \mathrm{~Hz}$ (thin solid line) and $1 \times 10^{9} \mathrm{~Hz}$ (dashed line) are shown. The minimum bandwidth is also shown (thick solid line).

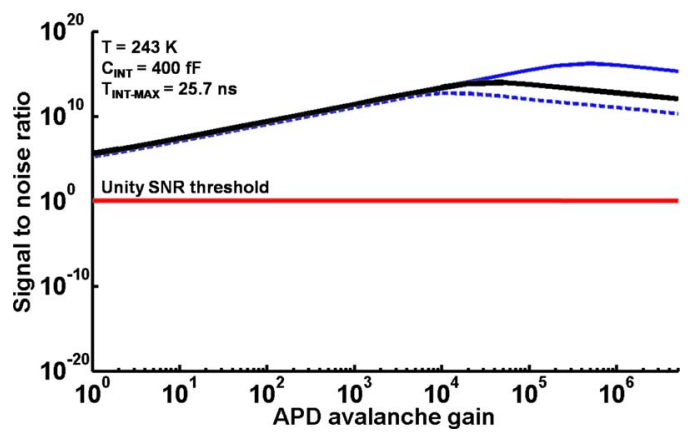

Fig. 16 SNR of the linear mode APD imager under AM 1.5 (97,000 lux) illumination. Bandwidths of $1 \times 10^{4} \mathrm{~Hz}$ (thin solid line) and $1 \times 10^{9} \mathrm{~Hz}$ (dashed line) are shown. The minimum bandwidth is also shown (thick solid line).

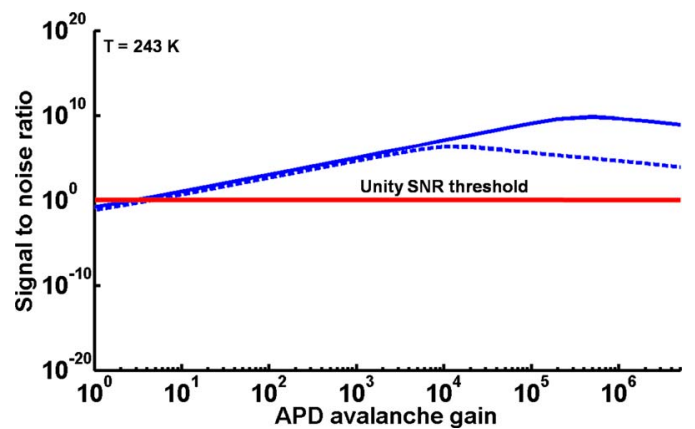

Fig. 17 SNR of the linear mode APD imager on a sunny day with 15,000 lux illumination. Bandwidths of $1 \times 10^{4} \mathrm{~Hz}$ (thin solid line) and $1 \times 10^{9} \mathrm{~Hz}$ (dashed line) are shown.

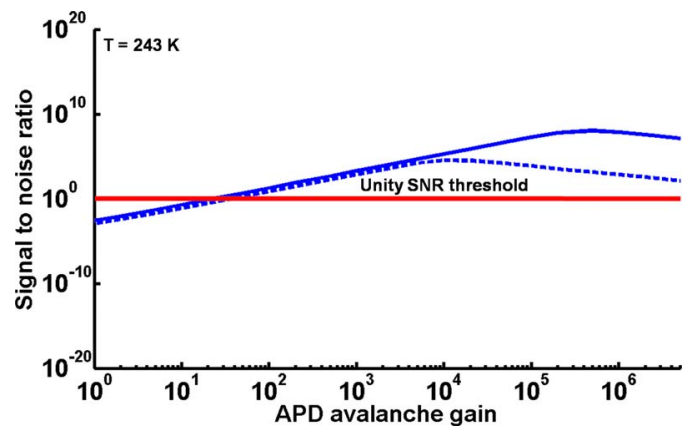

Fig. 18 SNR of the linear mode APD imager on a cloudy day with 2000 lux illumination. Bandwidths of $1 \times 10^{4} \mathrm{~Hz}$ (thin solid line) and $1 \times 10^{9} \mathrm{~Hz}$ (dashed line) are shown. 


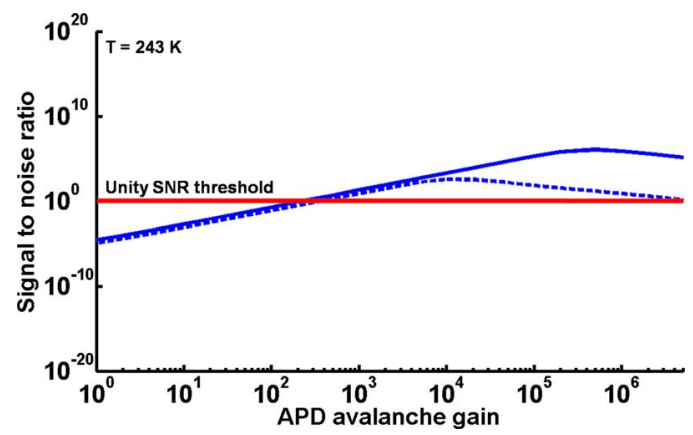

Fig. 19 SNR of the linear mode APD imager before dusk with 200 lux illumination. Bandwidths of $1 \times 10^{4} \mathrm{~Hz}$ (thin solid line) and $1 \times 10^{9} \mathrm{~Hz}$ (dashed line) are shown.

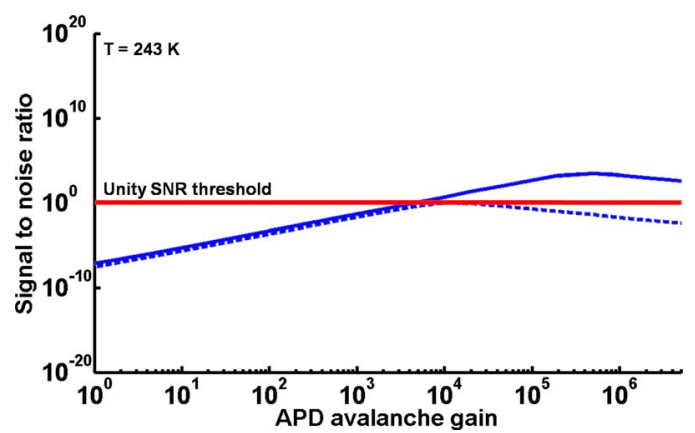

Fig. 20 SNR of the linear mode APD imager at dusk with 10 lux illumination. Bandwidths of $1 \times 10^{4} \mathrm{~Hz}$ (thin solid line) and $1 \times 10^{9} \mathrm{~Hz}$ (dashed line) are shown.

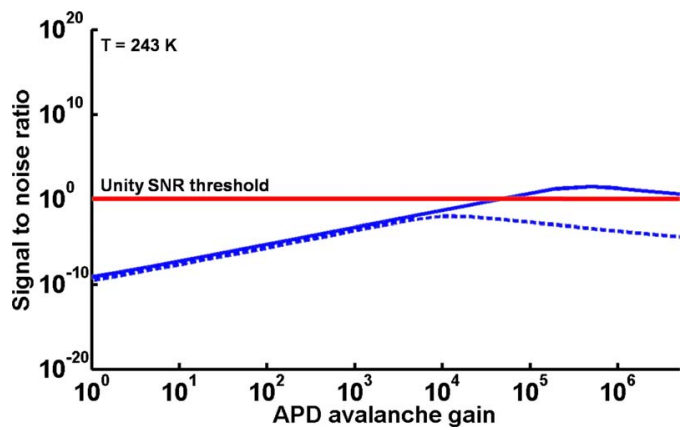

Fig. 21 SNR of the linear mode APD imager at late dusk with 1 lux illumination. Bandwidths of $1 \times 10^{4} \mathrm{~Hz}$ (thin solid line) and $1 \times 10^{9} \mathrm{~Hz}$ (dashed line) are shown.

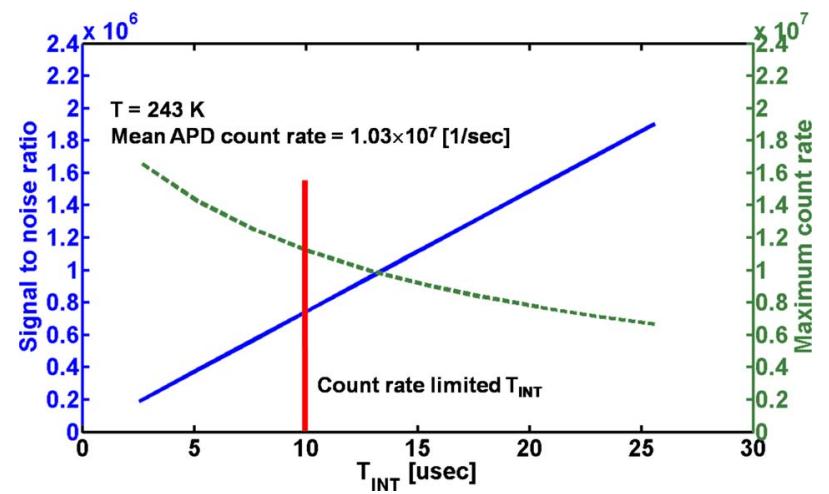

Fig. 22 SNR of the Geiger-mode APD imager at late dusk with 1 lux illumination. The Geiger-mode APD has 8-bit resolution and a quench time of $50 \mathrm{~ns}$.

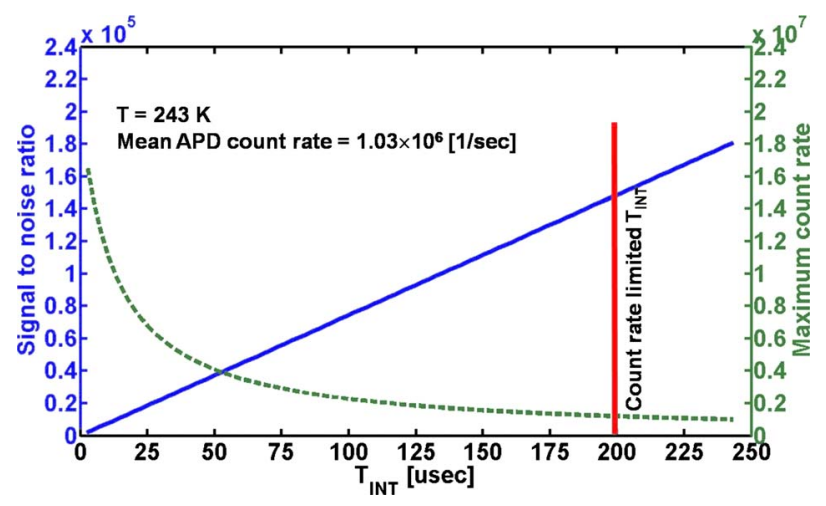

Fig. 23 SNR of the Geiger-mode APD imager on a clear night with full moon and 0.1 lux illumination. The Geiger-mode APD has 8-bit resolution and quench time of $50 \mathrm{~ns}$.

ity for imaging becomes limited, especially at larger bandwidths. After late dusk, however, the Geiger-mode operation of the $27 \mu \mathrm{m}$ mesa APD detector without a microlens is supported as calculated in Fig. 7.

The signal-to-noise ratio for the Geiger-mode mesa APD optical receiver that uses the APD in Fig. 1 and the electronic readout circuit in Fig. 10 is calculated using the expression given by Eq. (38).

$\mathrm{SNR}_{\mathrm{APD}-\text { Geiger-mode-receiver }}=\frac{\left\langle i_{p}(t)\right\rangle^{2}}{\sigma_{i_{p}}^{2}+\sigma_{i_{D}}^{2}}$.

In Eq. (38), the digital pixel control circuit does not add to the optical receiver noise. ${ }^{24,25}$ This enabling characteristic of the Geiger-mode pixel control circuit allows the optical receiver to operate with high sensitivity at the low light levels indicated in Fig. 7. Using the parameter values listed in Table 4, without the entries for the linear circuit, the signal-to-noise ratio for the wide dynamic range APD optical receiver in Geiger-mode is calculated according to Eq. (38). The results are shown in Figs. 22-26 for late dusk to a cloudy night with no moon.

In Fig. 22, the $27 \mu \mathrm{m}$ mesa APD pixel is shown to provide high sensitivity in the Geiger-mode. The SNR for $T_{\text {int }}=10 \mu \mathrm{sec}$ and with 8-bit resolution is $\sim 1 \times 10^{6}$ with 50,000 frames/sec.

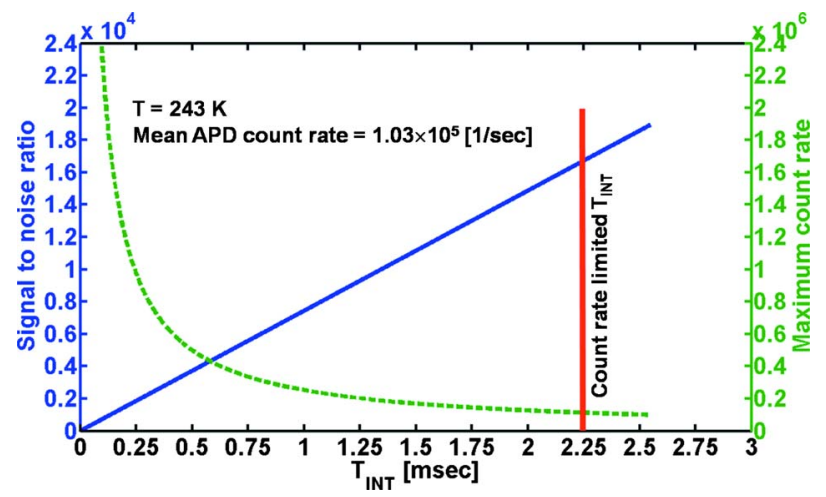

Fig. 24 SNR of the Geiger-mode APD imager on a clear night with quarter moon and 0.01 lux illumination. The Geiger-mode APD has 8-bit resolution and a quench time of $50 \mathrm{~ns}$. 


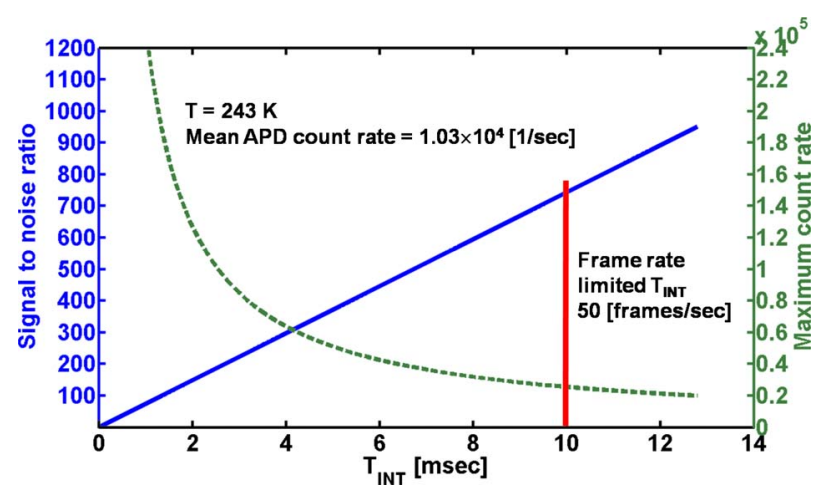

Fig. 25 SNR of the Geiger-mode APD imager in starlight with 0.001 lux illumination. The Geiger-mode APD has 8-bit resolution and a quench time of $50 \mathrm{~ns}$.

In Fig. 23, the SNR for $T_{\text {int }}=200 \mu$ sec and with 8-bit resolution is $\sim 1 \times 10^{5}$ with 2500 frames/sec.

In Fig. 24, the SNR for $T_{\text {int }}=2.25 \mathrm{msec}$ and with 8-bit resolution is $\sim 1 \times 10^{4}$ with 220 frames $/ \mathrm{sec}$.

In Fig. 25, the SNR for $T_{\text {int }}=10 \mathrm{msec}$ and with 8-bit resolution is $\sim 750$ with 50 frames/sec.

In Fig. 26, the SNR for $T_{\text {int }}=10 \mathrm{msec}$ and with 8-bit resolution is $\sim 7$ with 50 frames/sec. The calculations of signal-to-noise ratio for the $27 \mu \mathrm{m}$ wide dynamic range mesa APD optical receiver operating in Geiger-mode show that the imager will be capable of recording an image even under the lowest natural illumination conditions in Fig. 26. This result is important because it confirms that the detector array design should be capable of imaging successfully under the broadest range of natural illuminations when cooled only to $-30{ }^{\circ} \mathrm{C}$ using a compact two-stage thermoelectric cooler.

\section{Concluding Remarks}

It is shown in this detailed design study that a novel backilluminated silicon APD array architecture using (100) epitaxial silicon grown on $R$-plane sapphire having a latticematched AlN antireflective layer provides a unique means for creating high sensitivity, wide dynamic range APD device arrays for large area, high resolution, solid-state APDFPA imagers with zero optical crosstalk and 100\%

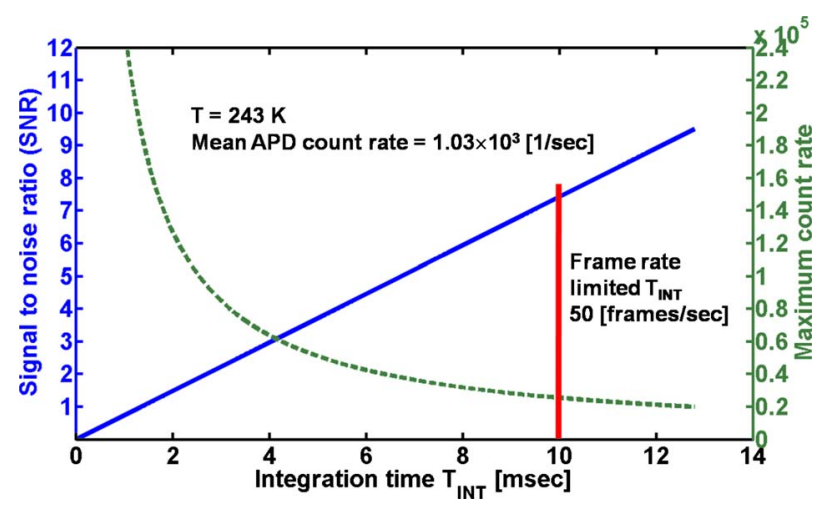

Fig. 26 SNR of the Geiger-mode APD imager on a cloudy night with no moon and 0.0001 lux illumination. The Geiger-mode APD has 8-bit resolution and a quench time of $50 \mathrm{~ns}$. sensitive-area-fill-factor. Such APD-FPA imagers could be used for both passive and active 2-D and 3-D imaging applications. The detailed calculations and analysis presented here show that the APD-FPA imager design will be capable of operating with high signal-to-noise ratio over the full range of natural illumination conditions near room temperature. Such a camera represents a novel, enabling technology for scientific and industrial applications.

\section{References}

1. A. G. Stern and D. C. Cole, "High quantum efficiency, backilluminated, crystallographically etched, silicon-on-sapphire avalanche photodiode with very wide dynamic range, for manufacturable high resolution imaging arrays" Proc. SPIE 7249, 72490R (2009).

2. A. G. Stern and D. C. Cole, "Design of a back-illuminated, crystallographically etched, silicon-on-sapphire avalanche photodiode with monolithically integrated microlens, for dual-mode passive and active arrays," Proc. SPIE 7153, $71530 Z$ (2008).

3. I. Rech, A. Ingargiola, R. Spinelli, I. Labanca, S. Marangoni, M. Ghioni, and S. Cova, "A new approach to optical crosstalk modelling in single-photon avalanche diodes," IEEE Photonics Technol. Lett. 20(50), 330-332 (2008).

4. S. Cihangir and S. Kwan, "Characterization of indium and solder bump bonding for pixel detectors," Nucl. Instrum. Methods Phys. Res. A 476(3), 670-675 (2002).

5. R. J. McIntyre "Multiplication noise in uniform avalanche diodes," IEEE Trans. Electron Devices 13(1), 164-168 (1966).

6. R. J. McIntyre, "A new look at impact ionization-part I: a theory of gain, noise, breakdown probability, and frequency response," IEEE Trans. Electron Devices 46(8), 1623-1631 (1999).

7. P. Yuan, K. A Anselm, C. Hu, H. Nie, C. Lenox, A. L. Holmes, B. G. Streetman, J. C. Campbell, and R. J. McIntyre, "A new look at impact ionization-part II: gain and noise in short avalanche photodiodes," IEEE Trans. Electron Devices 46(8), 1632-1639 (1999).

8. T. Shibata, K. Asai, Y. Nakamura, M. Tanaka, K. Kaigawa, J. Shibata, and H. Sakai, "AlN epitaxial growth on off-angle R-plane sapphire substrates by MOCVD," J. Cryst. Growth 229(1), 63-68 (2001).

9. W. Luo, X. Wang, L. Guo, H. Xiao, C. Wang, J. Ran, J. Li, and J. Li, "Influence of AIN buffer layer thickness on the properties of GaN epilayer on $\mathrm{Si}(111)$ by MOCVD," Microelectron. J. 39(12), 17101713 (2008)

10. Ü. Ozgür, G. Webb-Wood, H. O. Everitt, F. Yun, and H. Morkoc, "Systematic measurement of $\mathrm{Al}_{\mathrm{x}} \mathrm{Ga}_{1-\mathrm{x}} \mathrm{N}$ refractive indices," Appl. Phys. Lett. 79(25), 4103-4105 (2001).

11. C. K. Kim, "The physics of charge-coupled devices," in Charge Coupled Devices and Systems, M. J. Howes and D. V. Morgan, Eds., pp. 1-80, Wiley, New York (1979)

12. S. D. Donati, Photodetectors, Devices, Circuits and Applications, pp. 1-36, Prentice Hall, Upper Saddle River, NJ (2000).

13. C. Wehrli, "Extraterrestrial solar spectrum," Physikalisch Meteorologisches Observatorium-World Radiation Center (PMO/ WRC), Publication 615, Davos Dorf, Switzerland (1985).

14. L. W. Thomason, B. M. Herman, and J. A. Reagan, "The effect of atmospheric attenuators with structured vertical distributions on air mass determinations and Langley plot analyses," J. Atmos. Sci. 40, 1851-1854 (1983)

15. B. E. A. Saleh and M. C. Teich, Fundamentals of Photonics, pp. 246-253, 755, 789, John Wiley and Sons, New York (2007).

16. P. Kumar, P. Kwiat, A. Migdall, S. W. Nam, J. Vuckovic, and N. C Wong, "Photonic technologies for quantum information processing," Ouantum Inf. Process. 3(1-5), 215-231 (2004).

17. G. E. Stillman and C. M. Wolfe "Avalanche photodiodes" in Semiconductors and Semimetals, R. K. Willardson and A. C. Beer, Eds. pp. 291-391, Academic Press, New York (1977).

18. R. J. McIntyre, "The distribution of gains in uniformly multiplying avalanche photodiodes: theory," IEEE Trans. Electron Devices 19(6), 703-713 (1972)

19. J. C. Jackson, P. K. Hurley, B. Lane, and A. Mathewson, "Comparing leakage currents and dark count rates in Geiger-mode avalanche photodiodes," Appl. Phys. Lett. 80(22), 4100 (2002).

20. S. M. Sze, Physics of Semiconductor Devices, 2nd ed., pp. 495, 412 427, John Wiley and Sons, New York (1981).

21. A. Rohatgi and P. R. Choudhury, "Process induced effects on carrier lifetime and defects in float-zone silicon," J. Electrochem. Soc. 127(5), 1136-1139 (1980).

22. W. Zimmerman, "Experimental verification of the Shockley-Read Hall recombination theory in silicon," Electron. Lett. 9(16), 378-379 (1973).

23. D. A. Johns and K. Martin, Analog Integrated Circuit Design, pp. 181-217, John Wiley and Sons, New York (1996). 
24. M. Ghioni, S. Cova, F. Zappa, and C. Samori, "Compact active quenching circuit for fast counting with avalanche photodiodes," Rev. Sci. Instrum. 67(10), 3440-3448 (1996)

25. F. Zappa, M. Ghioni, S. Cova, C. Samori, and A. C. Giudice, "An integrated active-quenching circuit for single-photon avalanche diodes," IEEE Trans. Instrum. Meas. 49(6), 1167-1175 (2000).

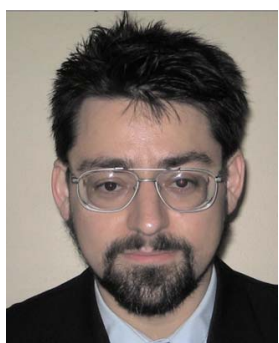

Alvin G. Stern recently completed his doctorate in electrical engineering at Boston University. He completed his BS degree in electrical engineering (BSEE) also at Boston University in May of 2000 . He was a member of the technical staff in the Advanced Imaging Technology Group at MIT Lincoln Laboratory for four years prior to returning to Boston University in 2004 to pursue $\mathrm{PhD}$ studies. He completed an MSEE in 2007 in the area of superconducting single photon detector (SSPD) characterization. Research interests include the design through analysis and simulation means of solid-state silicon avalanche photodiode (APD) devices and their arrays for imaging applications. Research interests further include the design of high-speed electronic readout circuits to control APD detector array operation.

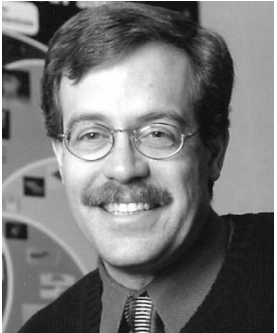

Daniel C. Cole is an associate professor in the Department of Mechanical Engineering at Boston University (BU). He obtained his doctorate in theoretical physics from the Graduate Center of the City University of New York in 1985. He worked for IBM Microelectronics in the area of physical simulation of semiconductor processes and microelectronic devices until joining $\mathrm{BU}$ in 1998. During this time he also wrote a number of articles involving the thermodynamics of charged particles and electromagnetic radiation. During the last three years at IBM, he managed a department of physicists, engineers, and mathematicians involved with developing and applying large computational programs to semiconductor problems. His present research work is split between the simulation of physical processes to aid the development of nanoelectronic devices, and the further investigation of an area of physics often called stochastic electrodynamics. 